\title{
An educational tool for the prophylaxis of hepatic encephalopathy
}

\author{
Maria Garrido, Matteo Turco, Chiara Formentin, Michela Corrias, Michele De Rui, \\ Sara Montagnese, Piero Amodio
}

To cite: Garrido M, Turco M, Formentin C, et al. An educational tool for the prophylaxis of hepatic encephalopathy. BMJ Open Gastro 2017;4:e000161. doi:10.1136/ bmjgast-2017-000161

Received 14 June 2017 Revised 16 July 2017 Accepted 25 July 2017

Check for updates

Department of Medicine, DIMED, University of Padova, Padova, Italy

Correspondence to Dr Sara Montagnese; sara.montagnese@unipd.it

\begin{abstract}
Background Providing structured information for the understanding of hepatic encephalopathy (HE) might be relevant to the prevention and management of the syndrome. The aim of our study was to design a brief, structured educational intervention and evaluate its usefulness in preventing $\mathrm{HE}$-related hospitalisation over time.

Methods Thirty-nine cirrhotic outpatients with a history of HE were enrolled and randomly assigned to an intervention (group $A ; n=20$ ) or control group (group $B ; n=19)$. All of them underwent evaluation of $\mathrm{HE}$ (clinical and quantitative neuropsychiatric assessment) and completed the Questionnaire on the Awareness of Encephalopathy. A 15 min educational session was then provided to patients in group $A$, including basic information on the pathophysiology, hygienic and medical management of $\mathrm{HE}$.

Results No demographic/clinical differences were observed at baseline between the two groups. Similarly, there were no significant differences in HE-related information available at baseline between the two groups; knowledge of HE was limited in both. The intervention was highly effective in increasing patients understanding of treatment of the condition (from $5 \%$ to $80 \%$ ). The educational intervention also reduced the risk of developing an episode of $\mathrm{HE}$ over a period of 12 months.

Conclusion The educational intervention confirmed the poor knowledge of patients with previous HE about their condition, served as a tool to increase patients' awareness, and minimised HE-related readmission rates over a period of 1 year.
\end{abstract}

\section{INTRODUCTION}

It has been estimated that the cumulative risk of recurrence after a first-ever episode of overt hepatic encephalopathy (HE) is nearly $40 \%$ over a period of 1 year. ${ }^{1}$ A retrospective analysis has reported that the readmission rate for HE-related reasons within 1 year is around $40 \% .^{2}$ The total healthcare cost for each HE hospitalisation has increased over the last decade, reaching \$37500 per each HE-related admission. ${ }^{3}$ Hence, preventing this complication of liver disease after discharge is challenging. ${ }^{4}$ The American Association
Summary box

What is already known about this subject?

- Patients with a history of hepatic encephalopathy (HE) and their caregivers have limited understanding of $\mathrm{HE}$ and its management.

What are the new findings?

- The HE educational procedure form (HEEPF), an educational intervention tool designed and tested in this study, increased patients' awareness and minimised HE-related readmission rates over a follow-up period of 1 year.

How might impact on clinical practice in the foreseeable future?

- This study provides pilot data to design and perform a definitive study on the usefulness of HEEPF.

for the Study of Liver Diseases/European Association for the Study of the Liver (AASLD/EASL) Practice Guideline ${ }^{4}$ has emphasised the importance of a multidisciplinary approach where patients, caregivers and practitioners should join to prevent/reduce the incidence of some precipitating factors of $\mathrm{HE}$, such as infections, constipation or dehydration, and avoid subsequent hospitalisations. ${ }^{4}$ The alliance of both patients and caregivers in the healthcare process has been proved to be effective in the reduction of hospital admissions in other diseases. ${ }^{56}$

In a recent study, we have observed that both patients and their caregivers have limited understanding of the disease/its management, and we have suggested that this may be the consequence of insufficient provision of information. ${ }^{7}$ Therefore, providing structured information for the understanding of HE might be relevant to its prevention and management. Based on this hypothesis, the aims of this study were: (1) to test the awareness of HE in a group of patients who had had a previous episode of HE $\geq$ grade II; (2) to validate a short educational procedure for 
these patients; (3) to provide a preliminary assessment of the efficacy, if any, of an educational intervention to prevent HE-related hospitalisation (HE $\geq$ grade II) over time by a prospective randomised intervention study.

\section{PATIENTS AND METHODS Participants}

A total of 39 consecutive outpatients referred to the Internal Medicine/Regional Centre for Liver Diseases of the Department of Medicine of Padova University Hospital were enrolled. The aetiology of cirrhosis was established based on clinical, laboratory, radiological, and histological indices. Its severity was determined using the Child-Pugh ${ }^{8}$ and Model for End-stage Liver Disease (MELD) $)^{9}$ scores. All patients had had at least one previous episode of overt HE ( $\geq$ grade II). ${ }^{10}$

Patients were excluded if they were under 18 years of age, had misused alcohol in the preceding 6 months, had a history of head injury, cardiovascular/cerebrovascular disease, neurological/psychiatric comorbidity, were taking psychoactive drugs or were unable/unwilling to comply with the study procedures. A total of 12 outpatients declared they had habitual caregivers who were relatives.

Experimental design and structured educational intervention The study was planned to be completed in 2 years and had a prospective randomised intervention design. Over this period of time, we were able to enrol 39 outpatients with cirrhosis, who were randomly assigned to one of two groups (by random numbers generated from a computer): group A, undergoing the educational intervention ( $n=20)$, and group $B$, not undergoing the educational intervention and serving as controls $(n=19)$.

Before starting the intervention, all participants underwent a neuropsychological and neurophysiological assessment (vide infra) and filled in the Questionnaire on the Awareness of Encephalopathy (QAE), which was designed by our research group in 2012 and is fully described elsewhere. ${ }^{7}$

The educational intervention was based on commenting three cartoons (HE educational procedure form (HEEPF)) including: (1) basic information on the pathophysiology of HE; (2) education on how to ensure/ maintain regular bowel emptying; and (3) drugs used to treat $\mathrm{HE}$ with a simple explanation of their mechanism of action, pharmacological and commercial names (figure 1). The educational intervention was planned to be completed in approximately $15 \mathrm{~min}$. The intervention was designed with a view to inform/educate the patient on manoeuvres and decisions that can be made without the intervention of a doctor, thus changes in the dose of diuretics and introduction/tapering of psychoactive drugs were not covered.

Within 1 hour of the educational intervention, patients completed a questionnaire designed to check if they were satisfied with the information received and if they had

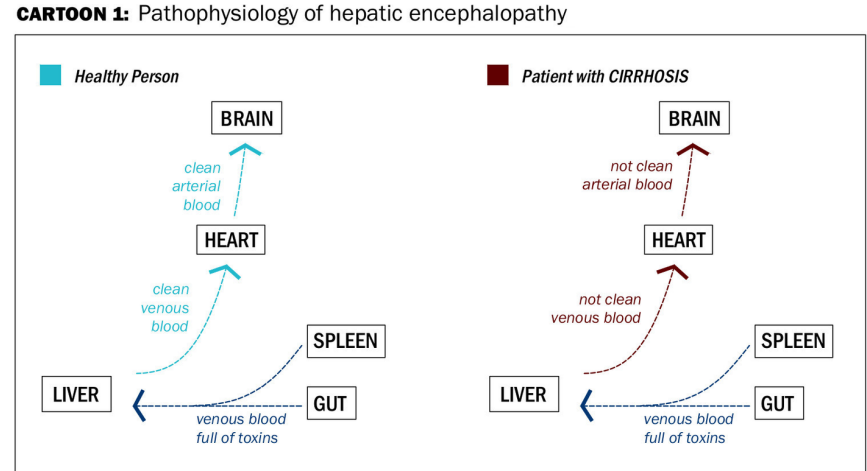

CARTOON 2: Hygienic measures to prevent hepatic encephalopathy

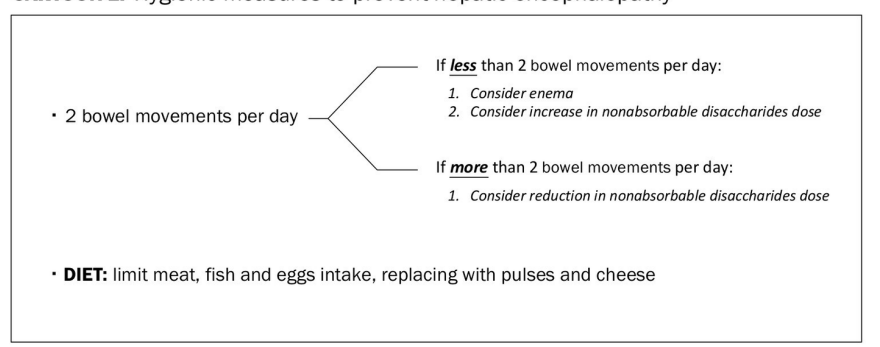

CARTOON 3: Drugs for hepatic encephalopathy
\begin{tabular}{|ll}
\hline Drugs reducing gut production of substances which are toxic for the brain \\
- LACTULOSE, LACTITOL (Laevolac, Portolac) & - PROBIOTICS (VSLAH, Yovis, etc) \\
- RIFAXIMIN (Normix) & - LEVOCARNITINE (Carnitene) \\
- PAROMOMYCIN SULFATE (Humatin) & - L-ORNITHINE L-ASPARTATE (Hepa-Merz) \\
- BRANCHED-CHAIN AMINOACIDS & - SODIUM BENZOATE \\
(AmInOram S, Friliver) & \\
* List of drugs available in Italy plus their commercial names
\end{tabular}

Figure 1 Hepatic encephalopathy educational procedure form.

acquired novel information (Novel Information Questionnaire (NIQ)) (table 1).

Both the HEEPF and the NIQ were designed for purposes of this specific study, and they are presented and described in table 1 and figure 1, respectively.

\section{Baseline neuropsychiatric assessment}

At the moment of recruitment, all the patients underwent a clinical assessment, including full neurological examination and a clinical grading of the neuropsychiatric abnormalities, if any, according to the West Haven criteria. $^{10}$

\section{Neuropsychological evaluation}

\section{Paper and pencil psychometry}

Psychometric performance was assessed, under standardised conditions, using Number Connection Tests A and B, the Digit Symbol subtest of the Wechsler Adult Intelligence Scale, and the Line Tracing and Serial Dotting tests. ${ }^{11}$ Individual test results were scored in relation to age-adjusted and education-adjusted Italian norms. ${ }^{12}$ Performance was classified as impaired if the rounded sum of the adjusted SD from the norms for the individual tests, known as the Psychometric Hepatic Encephalopathy Score (PHES), was $\leq-4{ }^{12}$ 
Table 1 Novel Information Questionnaire

1. Are you satisfied with the new information acquired on hepatic encephalopathy?

1.1. Please mark the correct statements on the causes of hepatic encephalopathy based on the information you have received

2. Are you satisfied with the information acquired on the procedures to prevent hepatic encephalopathy?

2.1. Please mark the correct statements on the prevention and treatment of hepatic encephalopathy based on the information you have received
Yes $\square$

No $\square$

The liver does not correctly clean venous blood $\square$

The brain does not receive cleaned arterial blood $\square$ The brain does not work properly $\square$

Yes $\square$

No $\square$

o bowel movements per day are necessary $\square$

If less than two bowel movements per day are obtained, you should administer a lactulose enema and/or increase the dose of lactulose/lactitol $\square$

If more than two bowel movements per day are obtained, you should consider reducing the dose of lactulose/lactitol $\square$

Non-absorbable antibiotics increase bowel movements $\square$

Non-absorbable antibiotics reduce the production of toxins in the gut $\square$

Branched chain amino acids increase bowel movements $\square$ Branched chain amino acids improve nutrition $\square$

\section{Sternberg task}

A computerised version of the Sternberg paradigm test was also administered. ${ }^{13}$ Thirty-six consecutive pairs of numbers, with or without common digits, were presented on a computer screen, and subjects were asked to press 1 on the keyboard if there were common digits (ie, 5632 and 694) and press 3 if there were no common digits (ie, 41 and 75). Both accuracy (\% correct responses) and reaction times (ms, adjusted for accuracy) were obtained. Finally, an overall age-adjusted and education-adjusted $\mathrm{z}$ score was obtained, with reference to local normative values.

\section{Neurophysiological evaluation}

Electroencephalograms (EEG) were recorded for $10 \mathrm{~min}$, eyes closed, in a condition of relaxed wakefulness, using a 21-electrode EEG cap (ground: Fpz; reference: Oz; impedance $<5 \mathrm{k}$ ). Each channel had its own analogue-todigital converter; the resolution was $0.19 \mu \mathrm{V} /$ bit (Brainquick 3200, Micromed, Mogliano Veneto, Italy). One continuous $80-100 \mathrm{~s}$ period of artefact-free EEG tracing was selected for spectral analysis; the following parameters were obtained: mean dominant frequency (MDF, expressed in $\mathrm{Hz}$ ), which is an estimate of the background frequency of the EEG, and relative power of the spectral bands delta, theta, alpha and beta, expressed as percentages $(\%)$. EEGs were classified according to Amodio et $a .^{14}$

On the day of study, patients were qualified as being neuropsychiatrically unimpaired (normal on clinical assessment, normal PHES and normal EEG) or as having covert HE (sgrade I on clinical assessment, ${ }^{10}$ abnormal PHES and/or EEG) or overt HE (>grade I on clinical assessment). ${ }^{10}$

\section{Follow-up}

Patients were telephoned monthly for 12 months, and they were asked if they had had HE-related admissions. Information was verified, where possible, on clinical notes and hospital records.

\section{Ethics}

The study was approved by the Padova University Hospital Ethics Committee, and all participants provided written, informed consent. The study was conducted according to the Declaration of Helsinki (Hong Kong Amendment) and Good Clinical Practice (European) guidelines.

\section{Statistical analysis}

Demographic, clinical and neuropsychiatric variables distributions were tested for normality using the Shapiro-Wilk's test. Differences between groups were performed by the Student's t-test/Mann-Whitney U test, as appropriate. Analysis of the effectiveness of the educational intervention was performed using the Fisher's exact test for proportions. Survival analysis was performed using both monovariate (Kaplan-Meier) and multivariate models (Cox's regression). Incidence was expressed as the number of events per year per 100 patients. The sum of the follow-up for each event to occur was computed. Then, the sum of the events and the sum of the observation time for the events to occur was computed. This value was normalised for 1 year and for 100 patients.

\section{RESULTS}

Demographic and baseline assessment parameters are presented in table 2 . There were no significant differences 
Table 2 Demographic, clinical, neuropsychological and EEG variables by group. No significant differences were observed between groups $A$ and $B$ in any of these variables

\begin{tabular}{|c|c|c|}
\hline & $\begin{array}{l}\text { Group A } \\
(n=20)\end{array}$ & $\begin{array}{l}\text { Group B } \\
(n=19)\end{array}$ \\
\hline Males (\%) & $14(70.0)$ & 17 (89.4) \\
\hline Age (years) & $64.1 \pm 8.8$ & $59.4 \pm 9.2$ \\
\hline Pugh's score & $7.1 \pm 1.3$ & $7.1 \pm 2.0$ \\
\hline \multicolumn{3}{|l|}{ Child (\%) } \\
\hline$A$ & 35 & 39 \\
\hline $\mathrm{B}$ & 65 & 50 \\
\hline $\mathrm{C}$ & 0 & 11 \\
\hline MELD score & $12.7 \pm 3.5$ & $12.3 \pm 3.9$ \\
\hline Overt HE* (\%) & 15 & 15.8 \\
\hline \multicolumn{3}{|l|}{ Aetiology (n (\%)) } \\
\hline Alcohol & $9(45)$ & $5(26)$ \\
\hline Viral & $8(40)$ & $6(31)$ \\
\hline Mixed & $2(10)$ & $4(21)$ \\
\hline Other & $1(5)$ & $4(21)$ \\
\hline \multicolumn{3}{|l|}{ Neuropsychological indices } \\
\hline MPZS & $-0.5 \pm 0.7$ & $-0.6 \pm 1.1$ \\
\hline PHES-z & $-1.7 \pm 2.3$ & $-2.6 \pm 4.1$ \\
\hline Abnormal PHES (n (\%)) & $4(21) \dagger$ & $6(31.5)$ \\
\hline $\begin{array}{l}\text { Sternberg reaction time } \\
\text { (ms) }\end{array}$ & $1713 \pm 307$ & $1728 \pm 472$ \\
\hline Sternberg accuracy (\%) & $75 \pm 23$ & $70 \pm 17$ \\
\hline Sternberg z-score & $-1.9 \pm 1.4$ & $-2.1 \pm 1.2$ \\
\hline \multicolumn{3}{|l|}{ Spectral EEG } \\
\hline MDF $(\mathrm{Hz})$ & $9 \pm 1$ & $9 \pm 2$ \\
\hline Delta (\%) & $8 \pm 5$ & $11 \pm 12$ \\
\hline Theta (\%) & $36 \pm 19$ & $41 \pm 16$ \\
\hline Alfa (\%) & $38 \pm 16$ & $30 \pm 14$ \\
\hline Beta (\%) & $18 \pm 9$ & $17 \pm 10$ \\
\hline
\end{tabular}

${ }^{*}$ Overt HE was graded according to the West Haven criteria (reference 10).

†PHES was not available for one patient.

EEG, electroencephalogram; HE, hepatic encephalopathy; MDF, mean dominant frequency; MELD, Model for End-stage Liver Disease; MPZS, mean psychometric z-score; PHES, Psychometric Hepatic Encephalopathy Score.

between groups $\mathrm{A}$ and $\mathrm{B}$ in any of the demographic, hepatic failure, neuropsychological and neurophysiological variables. Similarly, there were no significant differences between groups $\mathrm{A}$ and $\mathrm{B}$ in QAE results. In detail, 30 of the 39 patients $(77 \%, 95 \%$ CI 62 to 87$)$ were aware of previous HE, 16 in group A $(80 \%, 95 \%$ CI 58 to 92$)$ and 14 in group B (74\%, $95 \%$ CI 51 to 88$)$. However, only three patients in group A $(15 \%, 95 \%$ CI 5 to 36$)$ and two in group B (11\%, $95 \%$ CI 3 to 31$)$ were able to correctly identify the medication used to treat/ prevent HE. Furthermore, only one patient in group A $(5 \%, 95 \%$ CI 1 to 24$)$ and two in group B $(11 \%, 95 \%$ CI
3 to 31$)$ had adequate understanding of the expected treatment effects.

Twelve $(60 \%, 95 \%$ CI 39 to 78$)$ patients in group A declared they were satisfied with the HEEPF and 11 (55\%, $95 \%$ CI 34 to 74 ) had actually acquired novel information on the pathophysiology of HE. Of the eight $(40 \%$, 95\% CI 22 to 61 ) patients who declared that the intervention had not been useful to them, one $(5 \%, 95 \%$ CI 1 to 24) already had adequate information at baseline, five $(25 \%, 95 \%$ CI 11 to 47$)$ had not actually acquired new knowledge on their condition, and 2 (10\%, 95\% CI 3 to 30) had acquired new knowledge on their condition despite declaring the opposite. Thus, in 13 (65\%, 95\% CI 43 to 82 ) patients the intervention provided novel information and 14 (70\%, 95\% CI 48 to 85$)$ had adequate knowledge at the end of the educational procedure.

In relation to prevention/treatment of $\mathrm{HE}, 16$ patients (80\%, $95 \%$ CI 58 to 92 ) belonging to group A declared they had received novel information, and 4 (20\%, 95\% CI 8 to 42) declared they had not. Out of these four, one $(5 \%, 95 \%$ CI 1 to 24$)$ already had the information at baseline, and $3(15 \%, 95 \%$ CI 5 to 36$)$ had not actually acquired new information on prevention/treatment of HE, despite the HEEPF. Thus, the intervention was highly effective in increasing expertise on HE management, improving knowledge from 5\% (95\% CI 1 to 24) preintervention to $80 \%$ (95\% CI 58 to 92 ; p $<0.001$, Fisher's exact test) postintervention in group A.

During the 12-month follow-up period, patients in groups A and B suffered a total of 6 and 9 episodes of HE $\geq$ grade II requiring hospitalisation, respectively. The precipitating causes of HE were infection $(n=2)$, dehydration (1), constipation (1) and unknown (2) in group A, and infection (3), constipation (2), and unknown (4) in group B.

The incidence of events was $20.8 \times 100$ patients $\times$ year versus $48.8 \times 100$ patients $\times$ year in groups $A$ and $B$, respectively (figure 2). These results allow us to estimate that about 80 subjects would have been required to reach $80 \%$ power.

Older age, poor liver function and poor psychometric performance (abnormal PHES) were associated with increased risk of HE-related hospitalisation, while fast EEG activity was associated with reduced risk of HE-related hospitalisation (table 3).

By using multivariate backward Cox's model to adjust for these variables, which reflect the severity of the patients' clinical condition and may have acted as confounders, the educational intervention was shown to reduce the risk of HE-related hospitalisation over the follow-up period ( $\mathrm{HR}=0.14,95 \%$ CI 0.02 to $0.77, \mathrm{p}=0.02$ ).

\section{DISCUSSION}

A structured educational session (HEEPF) providing information on HE pathophysiology, prevention and medical treatment was designed, with a view to help preventing HE-related hospitalisations. The usefulness of 


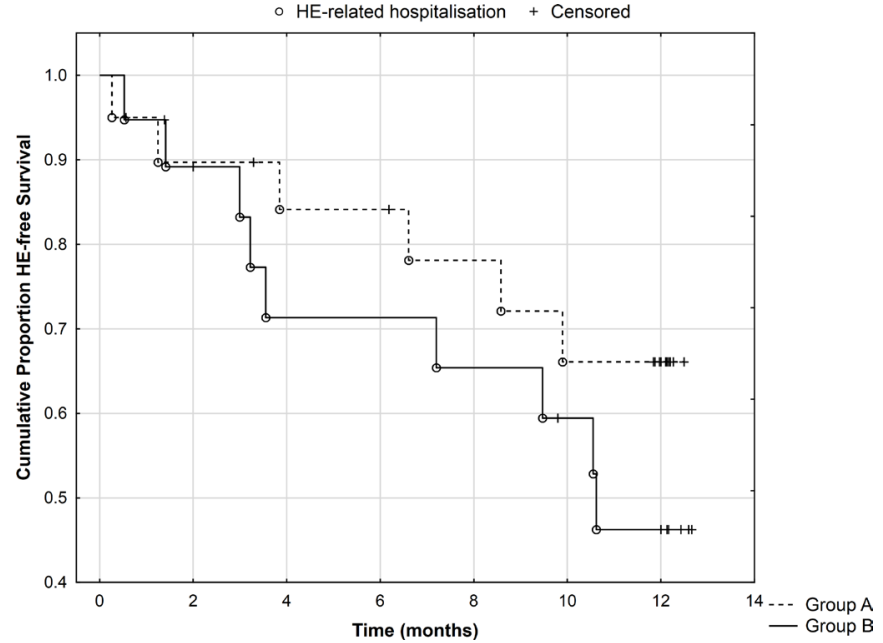

Figure 2 Cumulative proportion of HE-free patients over time by group ( $A=$ intervention; $B=$ no intervention). The risk of $\mathrm{HE}$-related hospitalisation in group $\mathrm{A}$ was $\mathrm{HR}=0.58(95 \% \mathrm{Cl}$ 0.21 to $1.62 ; p=0.29)$. HE, hepatic encephalopathy.

such programme was immediately evaluated by means of $\mathrm{NIQ}$, and over the subsequent 12 months by a monthly telephone interview, to check for the occurrence of HE-related hospitalisations.

After the intervention, $70 \%$ of patients had acquired new information about the pathophysiology of HE, and $80 \%$ of them had improved their knowledge about HE prevention/treatment. These findings highlight two issues: (1) the limited awareness of these patients about their condition, which is in line with a previous report ${ }^{7}$ and (2) the need to strengthen the communication between patients and physicians, at least within the context of the Italian healthcare system. In other diseases, educational approaches have been shown to enhance patients' personal understanding, to improve long-term control of the disease, and also to improve evidence of the usefulness of pharmacological treatment. ${ }^{15-18}$

Importantly, when the results were corrected for the severity of the clinical condition, HEEPF was shown to reduce the risk of HE recurrence over time. This was despite the high absolute number of HE events over the follow-up period. Although well-informed patients in group A could have avoided precipitant factors such as

Table 3 Monovariate analysis showing the association between age, liver function (MELD), abnormal PHES and EEG mean frequency (MDF) with the risk of developing HErelated hospitalisation

\begin{tabular}{lrll}
\hline Variables & Beta \pm SE & $\mathbf{p}$ & HR (95\% Cl) \\
\hline Age & $0.074 \pm 0.031$ & 0.016 & $1.08(1.01$ to 1.14$)$ \\
MELD & $0.141 \pm 0.066$ & 0.034 & $1.15(1.01$ to 1.31$)$ \\
Abnormal PHES & $1.035 \pm 0.544$ & 0.057 & $2.81(0.97$ to 8.17$)$ \\
MDF (EEG) & $-0.367 \pm 0.155$ & 0.017 & $0.69(0.51$ to 0.93$)$ \\
\hline
\end{tabular}

EEG, electroencephalogram; HE, hepatic encephalopathy; MDF, mean dominant frequency; MELD, Model for End-stage Liver Disease; PHES, Psychometric Hepatic Encephalopathy Score. constipation or dehydration, factors such as infection or bleeding are obviously more difficult to prevent/control. Nonetheless, the number of 'avoidable' precipitants in both groups was similar. The fact that the AASLD/EASL Practice Guideline on $\mathrm{HE}^{4}$ strongly encourages definition and recording of HE precipitants may help with similar studies in future, by way of clearer and better information in this respect in notes/medical records.

In addition, the AASLD/EASL Practice Guideline ${ }^{4}$ highlights the importance of the education of both patients and their relatives/caregivers. Caregivers have a crucial role in the healthcare process once the patient is discharged, ${ }^{4}$ hence new tools are being designed to help them detect episodes of HE ${ }^{19}$ Similarly, the HEEPF could have been administered to both patients and their caregivers, or even only to caregivers. Indeed, one of the initial aims of the present study was to involve caregivers. However, very few patients were accompanied on the day of study, making enrolment impossible.

Altogether, the improved awareness/understanding of $\mathrm{HE}$ by use of a single, quick ( $15 \mathrm{~min}$ ) educational intervention (HEEPF) constitutes preliminary evidence of its usefulness. The tool is cheap, user-friendly, and can be administered by trained nursing staff to improve patients' self-management (and possibly pharmacological treatment) so that HE-related readmission rates can be reduced. This has considerable consequences, as poor understanding of $\mathrm{HE} /$ its management among patients may also impinge on the likelihood of demonstrating the efficacy of treatments in future. In conclusion, the present study:

- confirms limited disease awareness in patients with a history of HE

- proposes a simple and short educational procedure (HEEPF) that is effective in increasing awareness, and most likely also in reducing readmission rates

- provides pilot data to design and perform a definitive study on the usefulness of HEEPF. Future research is also needed to characterise the features (ie, precipitants and others) of the HE with the highest likelihood of benefiting from the educational approach in terms of prevention.

Contributors MT, CF, MC, MDR and SM assessed the patients and performed the study. MG, CF, SM and PA analysed the data and drafted the manuscript. SM and PA designed and planned the study. SM is responsible for the overall content as guarantor.

Funding The work was partly funded by a grant from the Italian Ministry of Health to SM (Giovani Ricercatori 2009-1604309); MG was funded by a research postdoctoral fellowship from Gobierno de Extremadura (jointly financed by the European Regional Development Fund (ERDF); ref. P014013).

Competing interests None declared.

Patient consent Detail has been removed from this case description/these case descriptions to ensure anonymity. The editors and reviewers have seen the detailed information available and are satisfied that the information backs up the case the authors are making.

Ethics approval Padova University Hospital Ethics Committee.

Provenance and peer review Not commissioned; externally peer reviewed. 
Open Access This is an Open Access article distributed in accordance with the Creative Commons Attribution Non Commercial (CC BY-NC 4.0) license, which permits others to distribute, remix, adapt, build upon this work non-commercially, and license their derivative works on different terms, provided the original work is properly cited and the use is non-commercial. See: http://creativecommons.org/ licenses/by-nc/4.0/

(c) Article author(s) (or their employer(s) unless otherwise stated in the text of the article) 2017. All rights reserved. No commercial use is permitted unless otherwise expressly granted.

\section{REFERENCES}

1. Sharma BC, Sharma P, Agrawal A, et al. Secondary prophylaxis of hepatic encephalopathy: an open-label randomized controlled tria of lactulose versus placebo. Gastroenterology 2009;137:885-91.

2. Neff G, Barrett A, Graham CM, et al. Hospital costs, length of stay, and readmission rates in a cohort of cirrhotic patients discharged with hepatic encephalopathy. Hepatology 2013;58:390A-1.

3. Neff GW, Kemmer N, Duncan C, et al. Update on the management of cirrhosis - focus on cost-effective preventative strategies. Clinicoecon Outcomes Res 2013;5:143-52.

4. Vilstrup H, Amodio P, Bajaj J, et al. Hepatic encephalopathy in chronic liver disease: 2014 practice guideline by the American Association for the Study of Liver Diseases and the European Association for the Study of the Liver. Hepatology 2014:60:715-35.

5. Coleman EA, Williams MV. Executing high-quality care transitions: a call to do it right. J Hosp Med 2007;2:287-90.

6. Gleckman H. Coordinating care: an elusive but critical goal. Managing chronic illnesses during transitions presents tough challenges. Health Prog 2009;90:32-5.

7. Montagnese S, Amato E, Schiff S, et al. A patients' and caregivers' perspective on hepatic encephalopathy. Metab Brain Dis 2012;27:567-72.

8. Pugh RN, Murray-Lyon IM, Dawson JL, et al. Transection of the oesophagus for bleeding oesophageal varices. Br J Surg 1973;60:646-9.
9. Kamath PS, Wiesner RH, Malinchoc M, et al. A model to predict survival in patients with end-stage liver disease. Hepatology 2001;33:464-70.

10. Conn HO, Leevy CM, Vlahcevic ZR, et al. Comparison of lactulose and neomycin in the treatment of chronic portal-systemic encephalopathy. A double blind controlled trial. Gastroenterology 1977:72:573-83.

11. Weissenborn K, Ennen JC, Schomerus H, et al. Neuropsychological characterization of hepatic encephalopathy. $J$ Hepatol 2001;34:768-73.

12. Amodio P, Campagna F, Olianas $\mathrm{S}$, et al. Detection of minimal hepatic encephalopathy: normalization and optimization of the Psychometric Hepatic Encephalopathy Score. A neuropsychological and quantified EEG study. J Hepatol 2008:49:346-53.

13. Sternberg S. High-speed scanning in human memory. Science 1966;153:652-4.

14. Amodio P, Marchetti P, Del Piccolo F, et al. Spectral versus visual EEG analysis in mild hepatic encephalopathy. Clin Neurophysiol 1999;110:1334-44.

15. Hörnsten A, Stenlund H, Lundman B, et al. Improvements in HbA1c remain after 5 years--a follow up of an educational intervention focusing on patients' personal understandings of type 2 diabetes. Diabetes Res Clin Pract 2008;81:50-5.

16. Newman-Casey PA, Dayno M, Robin AL. Systematic review of educational interventions to improve glaucoma medication adherence: an update in 2015. Expert Rev Ophthalmol 2016;11:5-20.

17. Yamamoto T, Moyama S, Yano H. Effect of a newly-devised nutritional guide based on self-efficacy for patients with type 2 diabetes in Japan over 2 years: 1-year intervention and 1-year follow-up studies. J Diabetes Investig 2017;8:195-200.

18. Milazi M, Bonner A, Douglas C. Effectiveness of educational or behavioral interventions on adherence to phosphate control in adults receiving hemodialysis: a systematic review. JBI Database System Rev Implement Rep 2017;15:971-1010.

19. Bajaj JS, Frederick RT, Bass NM, et al. Overt hepatic encephalopathy: development of a novel clinician reported outcome tool and electronic caregiver diary. Metab Brain Dis 2016;31:1081-93. 